\title{
FUNDAMENTAL GROUP SCHEMES OF SOME QUOT SCHEMES ON A SMOOTH PROJECTIVE CURVE
}

\author{
CHANDRANANDAN GANGOPADHYAY AND RONNIE SEBASTIAN
}

\begin{abstract}
Let $k$ be an algebraically closed field. Let $C$ be an irreducible smooth projective curve over $k$. Let $E$ be a locally free sheaf on $C$ of rank $\geq 2$. Fix an integer $d \geq 2$. Let $\mathcal{Q}$ denote the Quot scheme parameterizing torsion quotients of $E$ of degree $d$. In this article we compute the $S$-fundamental group scheme of $\mathcal{Q}$.
\end{abstract}

\section{INTRODUCTION}

Let $X$ be a connected, reduced and complete scheme over a perfect field $k$ and let $x \in X$ be a $k$-rational point. In [Nor76], Nori introduced a $k$ group scheme $\pi^{N}(X, x)$ associated to essentially finite vector bundles on $X$. In [Nor82], Nori extends the definition of $\pi^{N}(X, x)$ to connected and reduced $k$-schemes. In [BPS06], Biswas, Parameswaran and Subramanian defined the notion of $S$-fundamental group scheme $\pi^{S}(X, x)$ for $X$ a smooth projective curve over any algebraically closed field $k$. This is generalized to higher dimensional connected smooth projective $k$-schemes and studied extensively by Langer in [Lan11, Lan12].

Let $C$ be a connected smooth projective curve defined over an algebraically closed field $k$. Fix a locally free sheaf $E$ on $C$ of rank $\geq 2$ and an integer $d \geq 2$. Let $\mathcal{Q}$ denote the Quot scheme parameterizing torsion quotients of $E$ of degree $d$. It is a smooth and projective variety over $k$. In this article we shall compute the $S$-fundamental group scheme of $\mathcal{Q}$. We mention some of the earlier results where fundamental group schemes were computed. In [BH15] it is proved that for a smooth projective surface $X$, the etale fundamental group $\pi^{\text {ét }}\left(H_{i l b_{X}^{n}}^{n}, n x\right)$ of the Hilbert scheme of $n$ points $(n \geq 2)$, is isomorphic to $\pi^{\text {ét }}(X, x)_{\mathrm{ab}}$. The main result in [PS19b] is to generalize this to the $S$-fundamental group scheme. In [Lan12] it is proved that $\pi^{S}(\operatorname{Alb}(C), 0)$ is isomorphic to $\pi^{S}(C, c)_{\mathrm{ab}}$. Let $S_{d}(d \geq 2)$ be the permutation group of $d$ symbols and denote $S^{d} C:=C^{d} / S_{d}$. In [PS19a] the authors prove that the $\pi^{S}\left(S^{d} C, d[c]\right)$ is isomorphic to $\pi^{S}(C, c)_{\mathrm{ab}}$. Once we have such a result for the $S$-fundamental group scheme, one deduces similar results for the Nori and etale fundamental group schemes.

Date: July 14, 2020.

2010 Mathematics Subject Classification. 14J60, 14F35, 14L15, 14 C05.

Key words and phrases. Finite vector bundle, $S$-fundamental group scheme, Hilbert scheme, semistable bundle, Tannakian category. 
Notation. From now on, unless mentioned otherwise, we will be working with the following assumptions. Let $k$ be an algebraically closed field. Let $C$ be an irreducible smooth projective curve over $k$. Let $E$ be a locally free sheaf on $C$ of rank $\geq 2$. Fix an integer $d \geq 2$. Let $\mathcal{Q}$ denote the Quot scheme parameterizing torsion quotients of $E$ of degree $d$. There is a Hilbert-Chow map $\phi: \mathcal{Q} \rightarrow S^{d} C$, the definition of which is recalled in section 2 .

The $S$-fundamental group scheme has been defined in Definition 7.1. The main result we prove in this article is the following.

Theorem (Theorem 7.4). For any closed point $q \in \mathcal{Q}$, there is an isomorphism of affine $k$-group schemes

$$
\phi_{*}^{S}: \pi^{S}(\mathcal{Q}, q) \stackrel{\sim}{\rightarrow} \pi^{S}\left(S^{d} C, \phi(q)\right) .
$$

From this the following corollary follows easily.

Corollary (Corollary 7.6). For any closed point $q \in \mathcal{Q}$, there are isomorphism of affine $k$-group schemes $\phi_{*}^{N}: \pi^{N}(\mathcal{Q}, q) \stackrel{\sim}{\rightarrow} \pi^{N}\left(S^{d} C, \phi(q)\right)$ and $\phi_{*}^{\text {ét }}: \pi^{\text {ét }}(\mathcal{Q}, q) \stackrel{\sim}{\longrightarrow} \pi^{\text {ét }}\left(S^{d} C, \phi(q)\right)$.

In view of $[\mathrm{PS} 19 \mathrm{a}]$ it follows that

Corollary 1.1. $\pi^{?}(\mathcal{Q}, q) \cong \pi^{?}(C, c)_{\mathrm{ab}}$ for $?=S, N$, ét.

The key ingredient in the proof of the above theorem is the following corollary regarding the scheme theoretic fiber of the Hilbert-Chow morphism. Related to this, in [BKR19, Proposition 5.9], the authors give a constructive proof that each fiber of the Hilbert-Chow map has the same reduction as the product of certain Quot schemes, which, a priori, need not be reduced. We prove the following. Let $D$ be a divisor corresponding to a closed point of $S^{d} C$. Let $\mathcal{Q}_{D}$ denote the scheme theoretic fiber over the point $D$. Then we have the following result.

Corollary (Corollary 6.6). The fiber $\mathcal{Q}_{D}$ is reduced, irreducible and normal.

Further, there is a smooth projective rational variety $S_{d}$ and a birational map $g_{d}: S_{d} \rightarrow \mathcal{Q}_{D}$, see Proposition 5.13. This allows us to conclude easily, using Grauert's theorem, that every numerically flat bundle is the pullback of a numerically flat along $\phi$.

In the rest of this article, $E$ will be a locally free sheaf of rank $\geq 2$ and $d$ will be an integer $\geq 2$.

Acknowledgements. We thank Arjun Paul for useful discussions. We thank the authors in [BKR19] for their interest. We thank the referee for a very careful reading of this article and for helpful comments.

\section{HilberT-CHOW MORPHISM}

In this section we recall the definition of the Hilbert Chow morphism $\phi: \mathcal{Q} \rightarrow S^{d} C$. For Hilbert schemes of points, one has a Hilbert-Chow morphism, see [FGA05, Chapter 7, section 1] for a detailed discussion. Here 
we describe how to get such a morphism for the Quot schemes we consider. This construction appears in other places, for example, see the introduction in [BDH15]. The map $\phi$ that we define here is the same as the map $\xi$ defined in [BKR19], in the situation that they work in. There, however, the authors give a more explicit description.

Let $p_{1}: C \times \mathcal{Q} \rightarrow C$ and $p_{2}: C \times \mathcal{Q} \rightarrow \mathcal{Q}$ denote the projections and let

$$
0 \rightarrow K \rightarrow p_{1}^{*} E \rightarrow B \rightarrow 0
$$

denote the universal quotient on $C \times \mathcal{Q}$. Since $B$ and $p_{1}^{*} E$ are flat over $\mathcal{Q}$, it follows that $K$ is a flat $\mathcal{Q}$ sheaf. Let $q \in \mathcal{Q}$ denote a closed point. Restricting this quotient to $C \times q$ we get the exact sequence

$$
\left.\left.0 \rightarrow K\right|_{C \times q} \rightarrow E \rightarrow B\right|_{C \times q} \rightarrow 0 .
$$

It follows that $\left.K\right|_{C \times q}$ is a locally free sheaf on $C$. From Nakayama's lemma it follows that $K$ is a locally free $C \times \mathcal{Q}$ sheaf of rank $r:=\operatorname{rank} E$. Taking determinant of the inclusion in (2.1) we get an exact sequence

$$
0 \rightarrow \operatorname{det}(K) \rightarrow \operatorname{det}\left(p_{1}^{*} E\right) \rightarrow \mathcal{F} \rightarrow 0 \text {. }
$$

To show that $\mathcal{F}$ is flat over $\mathcal{Q}$ it suffice to show that the restriction of this sequence to $C \times q$ remains exact on the left. But this is clear as the restriction of this sequence to $C \times q$ is precisely the sequence obtained by taking determinant of the inclusion in (2.2), which remains exact on the left. Thus, on $C \times \mathcal{Q}$ we get a quotient

$$
0 \rightarrow \operatorname{det}(K) \otimes \operatorname{det}\left(p_{1}^{*} E\right)^{-1} \rightarrow \mathcal{O} \rightarrow \mathcal{F} \otimes \operatorname{det}\left(p_{1}^{*} E\right)^{-1} \rightarrow 0 .
$$

This defines a morphism

$$
\phi: \mathcal{Q} \rightarrow S^{d} C .
$$

In the following sections we will study the fibers of this morphism.

\section{LOCUS WHERE $\phi$ IS SMOOTH}

Consider the map $\phi: \mathcal{Q} \rightarrow S^{d} C$. Let $D$ denote the divisor $\sum_{i=1}^{k} d_{i}\left[c_{i}\right]$ and consider a quotient $q$

$$
E \stackrel{q}{\rightarrow} \mathcal{O}_{D} \rightarrow 0
$$

Lemma 3.2. Given a quotient $q$ as above, there is a line bundle $L$ and a surjection $E \rightarrow L \rightarrow 0$ such that $q$ factors as

$$
E \rightarrow L \rightarrow \mathcal{O}_{D}
$$

Proof. Let $L^{\prime}$ be any line bundle on $C$. Then we have the exact sequence

$$
\left.0 \rightarrow L^{\prime}(-D) \rightarrow L^{\prime} \rightarrow L^{\prime}\right|_{D} \rightarrow 0 \text {. }
$$

Applying the functor $\operatorname{Hom}(E$,$) to the above exact sequence, we get$

$$
0 \rightarrow \operatorname{Hom}\left(E, L^{\prime}(-D)\right) \rightarrow \operatorname{Hom}\left(E, L^{\prime}\right) \rightarrow \operatorname{Hom}\left(E,\left.L^{\prime}\right|_{D}\right) \rightarrow \operatorname{Ext}^{1}\left(E, L^{\prime}(-D)\right)
$$


Now $\operatorname{Ext}^{1}\left(E, L^{\prime}(-D)\right) \cong H^{1}\left(E^{\vee} \otimes L^{\prime}(-D)\right)$, so for $L^{\prime}$ of sufficiently high degree we get $\operatorname{Ext}^{1}\left(E, L^{\prime}(-D)\right)=0$, that is, we have an exact sequence when $\operatorname{deg} L^{\prime} \gg 0$

$$
0 \rightarrow \operatorname{Hom}\left(E, L^{\prime}(-D)\right) \rightarrow \operatorname{Hom}\left(E, L^{\prime}\right) \rightarrow \operatorname{Hom}\left(E,\left.L^{\prime}\right|_{D}\right) \rightarrow 0 .
$$

In other words, for any homomorphism $\left.E \rightarrow L^{\prime}\right|_{D}$, we have a morphism $E \rightarrow L^{\prime}$ such that the following diagram commutes:



In particular, taking the quotient $q: E \rightarrow \mathcal{O}_{D}$, there is a line bundle $L^{\prime}$ such that $q$ factors as $E \rightarrow L^{\prime} \rightarrow \mathcal{O}_{D}$. Let $L$ be the image of $E$ in $L^{\prime}$. Then, we have a surjection

$$
E \rightarrow L \rightarrow \mathcal{O}_{D}
$$

which proves the lemma.

Lemma 3.3. The map $\phi: \mathcal{Q} \rightarrow S^{d} C$ is smooth at $q$ (corresponding to the quotient in equation (3.1)).

Proof. We will show that the map of Zariski tangent spaces $T_{q} \mathcal{Q} \rightarrow T_{\phi(q)} S^{d} C$ is surjective. Let $T:=\operatorname{Spec} k[\epsilon] /\left(\epsilon^{2}\right)$ and let $t_{0}$ denote the closed point of $T$. We will show that for any map

$$
T \stackrel{v}{\rightarrow} S^{d} C
$$

such that the image of the closed point of $T$ is $\phi(q)$, there is a map $T \stackrel{v^{\prime}}{\rightarrow} \mathcal{Q}$, such that the closed point maps to $q$ and the following diagram commutes

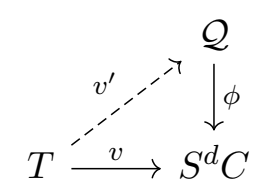

By universal property of $S^{d} C$, the morphism $v$ corresponds to a quotient over $C \times T$ given by

$$
\mathcal{O}_{C \times T} \stackrel{J_{v}}{\longrightarrow} \mathcal{O}_{\mathcal{D}} \rightarrow 0
$$

such that $\mathcal{O}_{\mathcal{D}}$ is $T$-flat and the restriction of $J_{v}$ to $C \times$ Spec $k$ is equivalent to the quotient $\left[\mathcal{O}_{C} \rightarrow \mathcal{O}_{D}\right]$ (which corresponds to the point $\phi(q)$ ). Note that $\mathcal{O}_{\mathcal{D}}$ is an Artinian ring.

Let $f_{1}: C \times T \rightarrow C$ and $f_{2}: C \times T \rightarrow T$ denote the projections. We fix a line bundle $L$ over $C$ as in Lemma 3.2. We have $1 \otimes J_{v}: f_{1}^{*} L \rightarrow f_{1}^{*} L \otimes \mathcal{O}_{\mathcal{D}}$. Define a quotient $J_{v^{\prime}}$ over $C \times T$ as the composition

$$
J_{v^{\prime}}: f_{1}^{*} E \rightarrow f_{1}^{*} L \stackrel{1 \otimes J_{v}}{\longrightarrow} f_{1}^{*} L \otimes \mathcal{O}_{\mathcal{D}} \cong \mathcal{O}_{\mathcal{D}} .
$$


Clearly, $f_{1}^{*} L \otimes \mathcal{O}_{\mathcal{D}}$ is $T$-flat and $\left.J_{v^{\prime}}\right|_{C \times\{\text { Spec } k\}}$ is equivalent to $q$ by Lemma 3.2. Hence, $J_{v^{\prime}}$ induces a morphism $v^{\prime}: T \rightarrow \mathcal{Q}$. Next we show that $\phi \circ v^{\prime}=v$. Let us denote the kernel of $J_{v^{\prime}}$ by $E_{v^{\prime}}$. Thus, we have an exact sequence

$$
0 \rightarrow E_{v^{\prime}} \stackrel{\iota}{\longrightarrow} f_{1}^{*} E \stackrel{J_{v^{\prime}}}{\longrightarrow} \mathcal{O}_{\mathcal{D}} \rightarrow 0 .
$$

Let $t_{0}$ denote the closed point of $T$. From the $T$-flatness of $\mathcal{O}_{\mathcal{D}}$ we conclude that $\left.E_{v^{\prime}}\right|_{C \times t_{0}}$ is locally free. Now using Nakayama's lemma we conclude that $E_{v^{\prime}}$ is locally free sheaf on $C \times T$. Recall from the definition of $\phi$, that the map $\phi \circ v^{\prime}$ is given by the following quotient on $C \times T$

$$
0 \rightarrow \operatorname{det}\left(E_{v^{\prime}}\right) \otimes \operatorname{det}\left(f_{1}^{*} E\right)^{-1} \stackrel{\operatorname{det}(\iota)}{\longrightarrow} \mathcal{O}_{C \times T} \rightarrow \mathcal{F} \rightarrow 0 .
$$

Passing to the local rings at $\left(c, t_{0}\right)$ and using equation (3.4), it is easily checked that $\mathcal{F} \cong \mathcal{O}_{\mathcal{D}}$ and that the above quotient is exactly $J_{v}: \mathcal{O}_{C \times T} \rightarrow$ $\mathcal{O}_{\mathcal{D}}$. This completes the proof of the lemma.

Proposition 3.5. In every fiber of $\phi$ there is a point at which $\phi$ is a smooth morphism.

Proof. Let $D$ be the divisor corresponding to a point $x \in S^{d} C$. Fix a line bundle $L$ which is a surjective quotient of $E$. Then the composite $q: E \rightarrow L \rightarrow L \otimes \mathcal{O}_{D}$ is a quotient such that $\phi(q)=x$. The proposition now follows from lemma 3.3.

\section{THE SPACE $S_{d}$}

Let the rank of the vector bundle $E$ be $r$. We will inductively define $\left(S_{d}, A_{d}\right)$, where $A_{d}$ is a vector bundle of rank $r$ defined over $C \times S_{d}$.

Define $S_{0}=$ Spec $k$ and $A_{0}=E$. To define $\left(S_{j}, A_{j}\right)$ we assume that we have defined $\left(S_{j-1}, A_{j-1}\right)$. Let $i_{j-1}:\{c\} \times S_{j-1} \hookrightarrow C \times S_{j-1}$ be the natural closed immersion. Define $S_{j}:=\mathbb{P}\left(i_{j-1}^{*} A_{j-1}\right)$ and let $f_{j, j-1}: S_{j} \rightarrow S_{j-1}$ be the structure morphism. Finally let $F_{j, j-1}:=i d_{C} \times f_{j, j-1}: C \times S_{j} \rightarrow$ $C \times S_{j-1}$. Let $p_{1, j}$ and $p_{2, j}$ be the projections from $C \times S_{j}$ to $C$ and $S_{j}$, respectively.

For each $j$, we have the following diagram

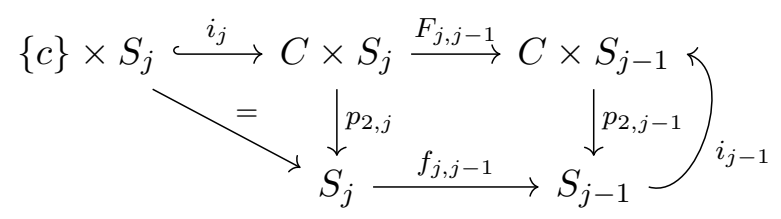

Let $\mathcal{O}_{j}(1)$ the universal line bundle over $S_{j}$. Then over $C \times S_{j}$ we have the quotient

$$
\begin{aligned}
F_{j, j-1}^{*} A_{j-1} & \rightarrow\left(i_{j}\right)_{*} i_{j}^{*} F_{j, j-1}^{*} A_{j-1} \\
& =\left(i_{j}\right)_{*} f_{j, j-1}^{*} i_{j-1}^{*} A_{j-1} \\
& \rightarrow\left(i_{j}\right)_{*} \mathcal{O}_{j}(1)
\end{aligned}
$$


Define $A_{j}$ to be the kernel of the above quotient. Since $\left(i_{j}\right)_{*} \mathcal{O}_{j}(1)$ is flat over $S_{j}$, restricting the exact sequence

$$
0 \rightarrow A_{j} \rightarrow F_{j, j-1}^{*} A_{j-1} \rightarrow\left(i_{j}\right)_{*} \mathcal{O}_{j}(1) \rightarrow 0
$$

to $C \times s$ we see that $\left.A_{j}\right|_{C \times s}$ is torsion free and so is locally free. It follows from Nakayama's lemma that $A_{j}$ is locally free on $C \times S_{j}$. Thus, we have defined $\left(S_{j}, A_{j}\right)$. It is easy to see, for example, using equation (5.2) in the proof of the next Lemma, that closed points of $S_{d}$ are in 1-1 correspondence with filtrations

$$
E_{d} \subset E_{d-1} \subset E_{d-2} \subset \cdots \subset E_{0}=E
$$

where each $E_{j}$ is a locally free sheaf of rank $r$ on $C$ and $E_{j} / E_{j+1}$ is a skyscraper sheaf of rank one supported at $c \in C$.

\section{Birationality of $S_{d}$ And $\mathcal{Q}_{d[c]}$}

Define the following morphisms for $j>i$ :

$$
\begin{aligned}
f_{j, i} & =f_{j, j-1} \circ \ldots \circ f_{i+1, i}: S_{j} \rightarrow S_{i}, \\
F_{j, i} & =F_{j, j-1} \circ \ldots \circ F_{i+1, i}: C \times S_{j} \rightarrow C \times S_{i} .
\end{aligned}
$$

Note that both of these morphisms are flat. Let $V \subset \mathcal{Q}_{d[c]}$ be the open subset whose points parameterize quotients of the type $E \rightarrow \mathcal{O}_{C} / \mathfrak{m}_{C, c}^{d}$.

Lemma 5.1. There exists a morphism $g_{d}: S_{d} \rightarrow \mathcal{Q}_{d[c]}$ such that

(i) $g_{d}$ is surjective on closed points,

(ii) $g_{d}^{-1}(V) \rightarrow V$ is a bijection,

(iii) $S_{d} \backslash g_{d}^{-1}(V) \rightarrow \mathcal{Q}_{d[c]} \backslash V$ has positive dimensional fibers.

Proof. We will define a quotient of $p_{1}^{*} E$ on $C \times S_{d}$. Using the flatness of $F_{d, i}$, we have inclusions (recall the definition of $A_{j}$ from (4.1))

$$
A_{d} \subset F_{d, d-1}^{*} A_{d-1} \subset F_{d, d-2}^{*} A_{d-2} \subset \ldots \subset F_{d, 1}^{*} A_{1} \subseteq p_{1}^{*} E .
$$

Define

$$
\begin{aligned}
B_{j}^{d} & :=p_{1}^{*} E / F_{d, j}^{*} A_{j} \\
& \cong F_{d, j}^{*}\left(p_{1}^{*} E / A_{j}\right)
\end{aligned}
$$

For each $j$ there is an exact sequence on $C \times S_{d}$

$$
0 \rightarrow F_{d, j-1}^{*} A_{j-1} / F_{d, j}^{*} A_{j} \rightarrow B_{j}^{d} \rightarrow B_{j-1}^{d} \rightarrow 0 .
$$

On $C \times S_{j}$ we have the quotient (4.1)

$$
0 \rightarrow A_{j} \rightarrow F_{j, j-1}^{*} A_{j-1} \rightarrow F_{j, j-1}^{*} A_{j-1} / A_{j} \cong\left(i_{j}\right)_{*}\left(\mathcal{O}_{j}(1)\right) \rightarrow 0 .
$$

As $F_{j, j-1}^{*} A_{j-1} / A_{j}$ is $S_{j}$-flat, the pullback along $F_{d, j}$, that is, $F_{d, j-1}^{*} A_{j-1} / F_{d, j}^{*} A_{j}$ is $S_{d}$-flat. When restricted to $C \times s$ for $s \in S_{d}$, it is a degree one torsion sheaf supported at $c$. By induction on $j$, using equation (5.3), one sees that 
$B_{j}^{d}$ is $S_{d}$-flat and the restriction of $B_{j}^{d}$ to $C \times s$ is a torsion sheaf of degree $j$ supported at $c$. In particular,

$$
0 \rightarrow A_{d} \rightarrow p_{1}^{*} E \rightarrow B_{d}^{d} \rightarrow 0
$$

is a quotient such that $B_{d}^{d}$ is $S_{d}$ flat and for each $s \in S_{d},\left.B_{d}^{d}\right|_{C \times\{s\}}$ is a torsion sheaf of degree $d$ supported at $c$. By the universal property of $\mathcal{Q}$, we have a morphism

$$
S_{d} \rightarrow \mathcal{Q},
$$

such that the set theoretic image of the composition

$$
S_{d} \rightarrow \mathcal{Q} \stackrel{\phi}{\rightarrow} S^{d} C
$$

is the point $d[c]$. Since $S_{d}$ is reduced, the scheme theoretic image is the scheme $\{d[c]\} \hookrightarrow S^{d} C$. In other words, we get that the above morphism factors as

$$
S_{d} \stackrel{g_{d}}{\longrightarrow} \mathcal{Q}_{d[c]} \longrightarrow \mathcal{Q}
$$

A closed point of $S_{d}$ corresponds to a filtration as in (4.2). Under $g_{d}$ this point maps to the quotient $E \rightarrow E / E_{d}$. Conversely, given a closed point of $\mathcal{Q}_{d[c]}$ it is clear that we can find a closed point of $S_{d}$ which maps to it. This proves (i). Suppose $E_{d} \subset E$ is such that $E / E_{d} \cong \mathcal{O} / \mathfrak{m}_{C, c}^{d}$, then for every $0 \leq j \leq d$ there is a unique $E_{j}$ such that $E_{d} \subset E_{j} \subset E$ and $E / E_{j} \cong \mathcal{O} / \mathfrak{m}_{C, c^{j}}^{j}$ From this one easily concludes (ii).

For a closed point in $\mathcal{Q}_{d[c]} \backslash V$, corresponding to a quotient $E \rightarrow \mathcal{F}_{d}$, we have $\operatorname{rank}\left(\mathcal{F}_{d} \otimes \mathcal{O} / \mathfrak{m}_{C, c}\right) \geq 2$. We can construct infinitely many chains $\mathcal{F}_{d} \rightarrow \mathcal{F}_{d-1} \rightarrow \ldots \rightarrow \mathcal{F}_{1}$. Therefore, the fiber over such a closed point is positive dimensional. This proves $($ iii).

Corollary 5.5. The fiber $\mathcal{Q}_{d[c]}$ is irreducible of dimension $d(r-1)$.

Proof. Since $S_{d}$ is irreducible, it is clear that $\mathcal{Q}_{d[c]}$ is irreducible. It is clear that the dimension of $S_{d}$ is $d(r-1)$. Thus, the dimension of $\mathcal{Q}_{d[c]}$ is at most $d(r-1)$. On the other hand, the dimension of the fiber of $\phi$ over a general point is $d(r-1)$. This shows that the dimension of $\mathcal{Q}_{d[c]}$ is at least $d(r-1)$.

Corollary 5.6. The codimension of $\mathcal{Q}_{d[c]} \backslash V$ in $\mathcal{Q}_{d[c]}$ is $\geq 2$.

Proof. As $S_{d}$ and $\mathcal{Q}_{d[c]}$ have the same dimension and $S_{d}$ is irreducible, this follows easily using (iii) in lemma 5.1.

Corollary 5.7. The fiber $\mathcal{Q}_{d[c]}$ satisfies Serre's condition $\left(R_{1}\right)$.

Proof. Since the map $\phi$ is smooth at points $v \in V$, it follows that $\mathcal{O}_{\mathcal{Q}_{d[c]}, v}$ is a regular local ring for all $v \in V$. Further, from the preceding corollary $V$ contains all prime ideals of height 1 . The corollary follows. 
Next we will show that $g_{d}$ is birational. Let

$$
p_{1}^{*} E \rightarrow B^{\prime}
$$

be the restriction of the universal quotient $B$ over $C \times \mathcal{Q}$ to the subscheme $C \times \mathcal{Q}_{d[c]}$. Let us define the inclusion

$$
i: \operatorname{Spec}\left(\mathcal{O}_{C, c} / \mathfrak{m}_{C, c}^{d}\right) \times \mathcal{Q}_{d[c]} \hookrightarrow C \times \mathcal{Q}_{d[c]} .
$$

Lemma 5.8. There is a coherent sheaf $F_{d}$ over $\operatorname{Spec}\left(\mathcal{O}_{C, c} / \mathfrak{m}_{C, c}^{d}\right) \times \mathcal{Q}_{d[c]}$ such that $B^{\prime}=i_{*} F_{d}$.

Proof. It is enough to show that the $p_{1}^{*}(E \otimes \mathcal{O}(-d c))$ is contained in the kernel of $p_{1}^{*} E \rightarrow B^{\prime}$. Denote the kernel by $A^{\prime}$. Let $0 \rightarrow E^{\prime} \stackrel{h}{\rightarrow} E$ be locally free sheaves of the same rank on a scheme $Y$. Let $\mathcal{I}$ denote the ideal sheaf determined by $\operatorname{det}(h)$. Then it is easy to see that $\mathcal{I} E \subset h\left(E^{\prime}\right) \subset E$. Thus, it suffices to find the ideal sheaf corresponding to the following exact sequence

$$
0 \rightarrow A^{\prime} \stackrel{h}{\rightarrow} p_{1}^{*} E \rightarrow B^{\prime} \rightarrow 0
$$

on $C \times \mathcal{Q}_{d[c]}$. By the definition of $\phi$, the map $\mathcal{Q}_{d[c]} \rightarrow \mathcal{Q} \stackrel{\phi}{\rightarrow} S^{d} C$ is given by the quotient

$$
0 \rightarrow \operatorname{det}\left(A^{\prime}\right) \stackrel{\operatorname{det}(h)}{\longrightarrow} \operatorname{det}\left(p_{1}^{*} E\right) \rightarrow \mathcal{F} \rightarrow 0
$$

on $C \times \mathcal{Q}_{d[c]}$. But since the image of $\mathcal{Q}_{d[c]}$ under this morphism is precisely $d[c]$, it follows that this quotient is isomorphic to the quotient

$$
p_{1}^{*} \mathcal{O}_{C} \rightarrow p_{1}^{*}\left(\mathcal{O}_{C} / \mathfrak{m}_{C, c}^{d}\right) .
$$

It is clear that the ideal sheaf $\mathcal{I}$ corresponding to the exact sequence (5.9) is $p_{1}^{*}\left(\mathcal{O}_{C}(-d c)\right)$. The lemma now follows.

Define subschemes

$$
D_{j}:=\operatorname{Spec}\left(\mathcal{O}_{C, c} / \mathfrak{m}_{C, c}^{j}\right) \times V \stackrel{\alpha_{j}}{\hookrightarrow} C \times V .
$$

By the previous lemma there is a sheaf $F_{d}$ on $D_{d}$ such that $\left.B^{\prime}\right|_{C \times V} \cong$ $\left(\alpha_{d}\right)_{*}\left(F_{d}\right)$. Clearly $F_{d}$ is flat over $V$ since $B^{\prime}$ is.

Lemma 5.10. $F_{d}$ is a line bundle over $D_{d}$.

Proof. By definition of $V$, for each $v \in V,\left(F_{d}\right)_{v} \cong \mathcal{O}_{C} / \mathfrak{m}_{C, c}^{d}$. Using $F_{d}$ is $V$-flat and Nakayama's lemma we see that $F_{d}$ is a line bundle.

Corollary 5.11. The restriction $F_{j}:=\left.F_{d}\right|_{D_{j}}$ is a line bundle on $D_{j}$.

Remark 5.12. We will use the following fact in the proof of the next theorem. Let $A$ and $B$ be rings and let $M$ be an $A \otimes_{k} B$ module. Let $B \rightarrow C$ be a ring homomorphism. Then

$$
M \otimes_{A \otimes_{k} B}\left(A \otimes_{k} C\right) \cong M \otimes_{A \otimes_{k} B}\left(A \otimes_{k} B\right) \otimes_{B} C \cong M \otimes_{B} C .
$$

In particular, if $0 \rightarrow N^{\prime} \rightarrow N \rightarrow M \rightarrow 0$ is a short exact sequence of $A \otimes_{k} B$ modules, where $M$ is flat as a $B$-module, then it remains exact when we apply the functor $-\otimes_{A \otimes_{k} B}\left(A \otimes_{k} C\right)$. 
Proposition 5.13. The restriction $g_{d}: g_{d}^{-1}(V) \rightarrow V$ is an isomorphism.

Proof. We will use induction on $j$ to define maps $V \rightarrow S_{j}$. Define $A_{0}^{\prime}$ on $C \times V$ to be $p_{1}^{*} E$. For $j \geq 1$ define sheaves $A_{j}^{\prime}$ on $C \times V$ as follows

$$
0 \rightarrow A_{j}^{\prime} \rightarrow p_{1}^{*} E \rightarrow\left(\alpha_{j}\right)_{*}\left(F_{j}\right) \rightarrow 0 .
$$

Observe that we have a commutative diagram

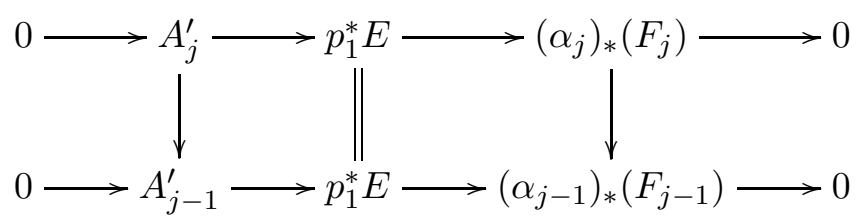

The kernel of the right vertical arrow is $\left(\alpha_{1}\right)_{*}\left(F_{1}\right)$. Thus, there is an exact sequence of sheaves on $C \times V$,

$$
0 \rightarrow A_{j}^{\prime} \rightarrow A_{j-1}^{\prime} \stackrel{\delta_{j-1}}{\longrightarrow}\left(\alpha_{1}\right)_{*}\left(F_{1}\right) \rightarrow 0 .
$$

Note that $S_{1}=\mathbb{P}\left(E_{c}\right)$. Thus, to give a map from $V \rightarrow S_{1}$ we need to give a line bundle quotient of $E_{c} \otimes O_{V}$. Restricting the universal quotient $p_{1}^{*} E \rightarrow\left(\alpha_{d}\right)_{*}\left(F_{d}\right)$ on $C \times V$ to $c \times V$ we get the quotient $E_{c} \otimes \mathcal{O}_{V} \rightarrow F_{1}$. This defines a morphism $h_{1}: V \rightarrow S_{1}$. On $C \times S_{1}$ one has the exact sequence (4.1). Using remark 5.12 we see that the pullback of this along $i d_{C} \times h_{1}$ gives the following exact sequence on $C \times V$,

$$
0 \rightarrow\left(i d_{C} \times h_{1}\right)^{*} A_{1} \rightarrow p_{1}^{*} E \stackrel{\delta_{0}}{\rightarrow}\left(\alpha_{1}\right)_{*}\left(F_{1}\right) \rightarrow 0 .
$$

We see that $A_{1}^{\prime}=\left(i d_{C} \times h_{1}\right)^{*} A_{1}$. Let us assume that we have constructed maps $h_{j-1}: V \rightarrow S_{j-1}$ such that the pullback of (4.1) along $i d_{C} \times h_{j-1}$ yields the exact sequence

$$
0 \rightarrow A_{j-1}^{\prime} \rightarrow A_{j-2}^{\prime} \stackrel{\delta_{j-2}}{\longrightarrow}\left(\alpha_{1}\right)_{*}\left(F_{1}\right) \rightarrow 0 .
$$

Consider the diagram

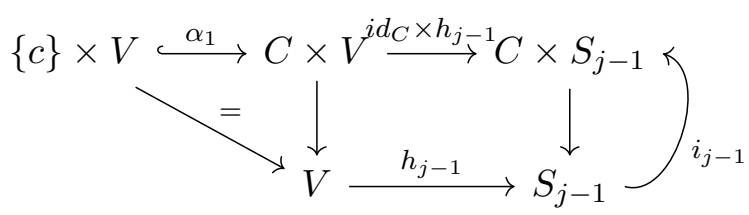

To give a map $V \rightarrow S_{j}$ we need to give a line bundle quotient of

$$
h_{j-1}^{*} i_{j-1}^{*} A_{j-1} \cong\left(\alpha_{1}\right)^{*}\left(i d_{C} \times h_{j-1}\right)^{*} A_{j-1} \cong\left(\alpha_{1}\right)^{*} A_{j-1}^{\prime},
$$

where the last isomorphism follows from (5.16). Restricting (5.15) to $c \times V$, we get a line bundle quotient

$$
\left(\alpha_{1}\right)^{*} A_{j-1}^{\prime} \rightarrow F_{1}
$$


This defines a morphism $h_{j}: V \rightarrow S_{j}$. Pulling back (4.1) along $i d_{C} \times h_{j}$, using 5.12 and (5.16), we get the following short exact sequence on $C \times V$

$$
0 \rightarrow\left(i d_{C} \times h_{j}\right)^{*} A_{j} \rightarrow A_{j-1}^{\prime} \stackrel{\delta_{j-1}}{\longrightarrow}\left(\alpha_{1}\right)_{*}\left(F_{1}\right) \rightarrow 0 .
$$

Now equation (5.15) shows that $A_{j}^{\prime} \cong\left(i d_{C} \times h_{j}\right)^{*} A_{j}$ and there is an exact sequence

$$
0 \rightarrow A_{j}^{\prime} \rightarrow A_{j-1}^{\prime} \stackrel{\delta_{j-1}}{\longrightarrow}\left(\alpha_{1}\right)_{*}\left(F_{1}\right) \rightarrow 0
$$

Thus, inductively we have constructed a map $h_{d}: V \rightarrow S_{d}$.

To show that the composite $V \stackrel{h_{d}}{\longrightarrow} S_{d} \stackrel{g_{d}}{\longrightarrow} \mathcal{Q}_{d[c]}$ is an isomorphism onto $V$, it suffices to show that the pullback of the universal quotient on $C \times \mathcal{Q}_{d[c]}$ along $i d_{C} \times\left(g_{d} \circ h_{d}\right)$ is the restriction of the universal quotient to $C \times V$. Recall from (5.14) the universal quotient on $C \times V$ is

$$
0 \rightarrow A_{d}^{\prime} \rightarrow p_{1}^{*} E \rightarrow B^{\prime} \rightarrow 0 .
$$

Pulling this back along $i d_{C} \times g_{d}$ is the quotient (recall from (5.4))

$$
0 \rightarrow A_{d} \rightarrow p_{1}^{*} E \rightarrow B_{d}^{d} \rightarrow 0,
$$

by the definition of the map $g_{d}$. From the definition of $h_{d}$, one easily checks that the pullback along $i d_{C} \times h_{d}$ of the filtration in (5.2) is the following filtration on $C \times V$,

$$
A_{d}^{\prime} \subset A_{d-1}^{\prime} \subset \cdots \subset p_{1}^{*} E .
$$

Thus, it follows that the pullback along $i d_{C} \times h_{d}$ of (5.4) is

$$
0 \rightarrow A_{d}^{\prime} \rightarrow p_{1}^{*} E \rightarrow B^{\prime} \rightarrow 0,
$$

which is the universal quotient on $C \times V$. This proves that $g_{d} \circ h_{d}$ is the identity on $V$. By lemma 3.3 the morphism $\phi$ is smooth at a point $v \in V$. This shows that the local ring $\mathcal{O}_{V, v}$ is a domain. Thus, we have maps $\mathcal{O}_{V, v} \rightarrow \mathcal{O}_{S_{d}, h_{d}(v)} \rightarrow \mathcal{O}_{V, v}$ such that the composite is the identity. Since both rings have the same dimension, the kernel of $\mathcal{O}_{S_{d}, h_{d}(v)} \rightarrow \mathcal{O}_{V, v}$ is forced to be 0 , which shows that the local rings are isomorphic. This proves the proposition.

\section{Normality OF ALL FIBERS}

For a point $D=\sum d_{i}\left[c_{i}\right] \in S^{d} C$, denote by $\mathcal{Q}_{D}$ the scheme theoretic fiber of $\phi$ over the closed point corresponding to $D$.

Proposition 6.1. The fiber $\mathcal{Q}_{D}$ is irreducible of dimension $d(r-1)$.

Proof. We define a morphism $\prod \mathcal{Q}_{d_{i}\left[c_{i}\right]} \rightarrow \mathcal{Q}_{D}$ as follows. Let $p_{j}$ be the projections $C \times \prod \mathcal{Q}_{d_{i}\left[c_{i}\right]} \rightarrow C \times \mathcal{Q}_{d_{j}\left[c_{j}\right]}$ and $p$ be the projection $C \times \prod \mathcal{Q}_{d_{i}\left[c_{i}\right]} \rightarrow$ $C$. Let $B_{d_{i}\left[c_{i}\right]}$ denote the universal quotient over $C \times \mathcal{Q}_{d_{i}\left[c_{i}\right]}$. Then over $C \times \prod \mathcal{Q}_{d_{i}\left[c_{i}\right]}$, we define a quotient

$$
p^{*} E \rightarrow \bigoplus p_{i}^{*} B_{d_{i}\left[c_{i}\right]}
$$


Clearly, $\bigoplus p_{i}^{*} B_{d_{i}\left[c_{i}\right]}$ is flat, and hence induces a morphism

$$
\theta_{D}: \prod \mathcal{Q}_{d_{i}\left[c_{i}\right]} \rightarrow \mathcal{Q}
$$

which is bijective onto the closed points of $\mathcal{Q}_{D}$. Therefore, $\mathcal{Q}_{D}$ is irreducible. Since the dimension of the general fiber of $\phi$ is $d(r-1)$, we get

$$
d(r-1) \leq \operatorname{dim} \mathcal{Q}_{D} \leq \sum \operatorname{dim} \mathcal{Q}_{d_{i}\left[c_{i}\right]}=\sum d_{i}(r-1)=d(r-1)
$$

This proves the corollary.

Corollary 6.3. The map $\phi$ is flat.

Proof. This follows using [Har77, Chapter III, Exercise 10.9]

Corollary 6.4. The fiber $\mathcal{Q}_{d[c]}$ is reduced, irreducible and normal. In particular, it is integral.

Proof. Since $\phi$ is flat and $\mathcal{Q}$ is smooth, it follows from [Stk, Tag 045J] (or see Corollary to [Mat86, Theorem 23.3]) that the fiber $\mathcal{Q}_{d[c]}$ is Cohen-Macaulay. Thus, the fiber satisfies Serre's condition $\left(S_{2}\right)$. Now from corollary 5.7 it follows that the fiber satisfies $\left(R_{0}\right)$ and $\left(S_{1}\right)$ and so it is reduced. Since it satisfies $\left(R_{1}\right)$ and $\left(S_{2}\right)$ it is normal.

Lemma 6.5. $\mathcal{Q}_{D} \cong \prod \mathcal{Q}_{d_{i}\left[c_{i}\right]}$.

Proof. The map $\theta_{D}$ in (6.2) sits in a commutative diagram

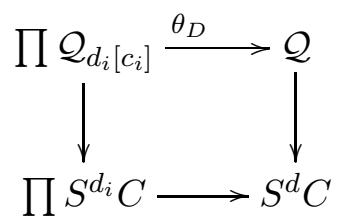

From the above diagram it is clear that $\theta_{D}$ factors to give a map

$$
\tilde{\theta}_{D}: \prod \mathcal{Q}_{d_{i}\left[c_{i}\right]} \rightarrow \mathcal{Q}_{D}
$$

We want to give a map in the other direction. Let $p_{D}: C \times \mathcal{Q}_{D} \rightarrow C$ be the first projection. Let us denote the restriction of the universal quotient to $C \times \mathcal{Q}_{D}$ by

$$
p_{D}^{*} E \rightarrow B_{D} .
$$

There are integers $e_{i}$ such that the quotient $B_{D}$ is supported on the following closed subscheme of $C \times \mathcal{Q}_{D}$

$$
\bigsqcup_{i} \operatorname{Spec}\left(\mathcal{O}_{C} / \mathfrak{m}_{C, c_{i}}^{e_{i}}\right) \times \mathcal{Q}_{D}
$$

Let $\mathfrak{j}_{i}: \operatorname{Spec}\left(\mathcal{O}_{C} / \mathfrak{m}_{C, c_{i}}^{e_{i}}\right) \times \mathcal{Q}_{D} \hookrightarrow C \times \mathcal{Q}_{D}$ denote the closed immersion. Let

$$
B_{d_{i}\left[c_{i}\right]}:=\mathfrak{j}_{i *}\left(\left.B_{D}\right|_{\operatorname{Spec}\left(\mathcal{O}_{C} / \mathfrak{m}_{C, c_{i}}^{e_{i}}\right) \times \mathcal{Q}_{D}}\right) .
$$


Clearly, since $B_{D}$ is flat over $\mathcal{Q}_{D}$, the $B_{d_{i}\left[c_{i}\right]}$ is also flat over $\mathcal{Q}_{D}$. We define the quotients

$$
p^{*} E \rightarrow B_{D} \rightarrow B_{d_{i}\left[c_{i}\right]},
$$

which defines a morphism $\mathcal{Q}_{D} \rightarrow \mathcal{Q}_{d_{i}\left[c_{i}\right]}$. This defines a morphism $\gamma_{D}$ : $\mathcal{Q}_{D} \rightarrow \prod \mathcal{Q}_{d_{i}\left[c_{i}\right]}$. One easily checks that the pullback along $i d_{C} \times\left(\theta_{D} \circ \gamma_{D}\right)$ of the universal quotient is $p_{1}^{*} E \rightarrow B_{D}$. This shows that $\tilde{\theta}_{D} \circ \gamma_{D}$ is the identity. Arguing as in the last para of the proof of proposition 5.13, the lemma is proved.

Corollary 6.6. The fiber $\mathcal{Q}_{D}$ is reduced, irreducible and normal.

\section{Main TheOREM}

Definition 7.1. Let $X$ be a connected, projective and reduced $k$-scheme. Let $\mathcal{C}^{\mathrm{nf}}(X)$ denote the full subcategory of coherent sheaves whose objects are coherent sheaves $E$ on $X$ satisfying the following two conditions:

(1) $E$ is locally free, and

(2) for any smooth projective curve $C$ over $k$ and any morphism $f$ : $C \longrightarrow X$, the vector bundle $f^{*} E$ is semistable of degree 0 .

We call the objects of the category $\mathcal{C}^{\mathrm{nf}}(X)$ numerically flat vector bundles on $X$. Fix a $k$-valued point $x \in X$. Let $\operatorname{Vect}_{k}$ be the category of finite dimensional $k$-vector spaces. Let $T_{x}: \mathcal{C}^{\text {nf }}(X) \longrightarrow$ Vect $_{k}$ be the fiber functor defined by sending an object $E$ of $\mathcal{C}^{\text {nf }}(X)$ to its fiber $E_{x} \in$ Vect $_{k}$ at $x$. Then $\left(\mathcal{C}^{\mathrm{nf}}(X), \otimes, T_{x}, \mathcal{O}_{X}\right)$ is a neutral Tannaka category [Lan11, Proposition 5.5, p. 2096]. The affine $k$-group scheme $\pi^{S}(X, x)$ Tannaka dual to this category is called the $S$-fundamental group scheme of $X$ with base point $x$ [Lan11, Definition 6.1, p. 2097].

A vector bundle $E$ is said to be finite if there are distinct non-zero polynomials $f, g \in \mathbb{Z}[t]$ with non-negative coefficients such that $f(E) \cong g(E)$.

Definition 7.2. A vector bundle $E$ on $X$ is said to be essentially finite if there exist two numerically flat vector bundles $V_{1}, V_{2}$ and finitely many finite vector bundles $F_{1}, \ldots, F_{n}$ on $X$ with $V_{2} \subseteq V_{1} \subseteq \bigoplus_{i=1}^{n} F_{i}$ such that $E \cong V_{1} / V_{2}$.

Let $\operatorname{EF}(X)$ be the full subcategory of coherent sheaves whose objects are essentially finite vector bundles on $X$. Fix a closed point $x \in X$ and let $T_{x}: \mathrm{EF}(X) \longrightarrow \operatorname{Vect}_{k}$ be the fiber functor defined by sending an object $E \in \operatorname{EF}(X)$ to its fiber $E_{x}$ at $x$. Then the quadruple $\left(\mathrm{EF}(X), \otimes, T_{x}, \mathcal{O}_{X}\right)$ is a neutral Tannakian category. The affine $k$-group scheme $\pi^{N}(X, x)$ Tannaka dual to this category is referred to as the Nori-fundamental group scheme of $X$ with base point $x$, see [Nor76] for more details.

In [Lan11, Proposition 8.2] it is proved that the $S$-fundamental group of projective space is trivial. In [HM11] it is proved that the $S$-fundamental group scheme is a birational invariant of smooth projective varieties. 
Let $S_{d_{i}, c_{i}}$ be the space defined in section 4 by taking $d=d_{i}$ and $c=c_{i}$. In view of the discussion in section 5 there is a birational map

$$
\eta_{D}:=\left(\tilde{\theta}_{D} \circ \prod g_{d_{i}}\right): \prod S_{d_{i}, c_{i}} \rightarrow \prod \mathcal{Q}_{d_{i}\left[c_{i}\right]} \rightarrow \mathcal{Q}_{D} .
$$

Proposition 7.3. A numerically flat bundle on $\mathcal{Q}_{D}$ is trivial.

Proof. Let $W$ be a numerically flat bundle on $\mathcal{Q}_{D}$. As $\mathcal{Q}_{D}$ is normal, $\eta_{D}$ is birational, $\prod S_{d_{i}, c_{i}}$ is a smooth rational variety, we have

$$
\begin{aligned}
W & \cong \eta_{D *} \eta_{D}^{*} W \\
& \cong \eta_{D *}(\mathcal{O})^{\oplus r} \\
& \cong \mathcal{O}_{\mathcal{Q}_{D}}^{\oplus r}
\end{aligned}
$$

In the above we have used the result of [HM11]. This proves the proposition.

We now prove the main result of this article.

Theorem 7.4. Let $k$ be an algebraically closed field. Let $C$ be an irreducible smooth projective curve over $k$. Let $E$ be a locally free sheaf on $C$ of rank $\geq 2$. Fix an integer $d \geq 2$. Let $\mathcal{Q}$ denote the Quot scheme parameterizing torsion quotients of $E$ of degree $d$. Let $S^{d} C$ denote the dth symmetric product of $C$ and let $\phi: \mathcal{Q} \rightarrow S^{d} C$ denote the Hilbert Chow map (see section 2). Then the induced map $\phi_{*}^{S}: \pi^{S}(\mathcal{Q}, q) \rightarrow \pi^{S}\left(S^{d} C, \phi(q)\right)$ is an isomorphism.

Proof. Since the fibers of $\phi$ are projective integral varieties, and $\phi$ is flat, it follows that $\phi_{*}\left(\mathcal{O}_{\mathcal{Q}_{D}}\right)=\mathcal{O}_{S^{d} C}$. Now applying [Lan11, Lemma 8.1] we see that $\phi_{*}^{S}$ is faithfully flat. To prove $\phi_{*}^{S}$ is a closed immersion we will use [DMOS82, Proposition 2.21(b)], which we recall for the convenience of the reader. For an affine algebraic group scheme $G$ over $k$, let $\operatorname{Rep}_{k}(G)$ denote the category of finite dimensional representations of $G$ on $k$-vector spaces. Let $\theta: G \rightarrow G^{\prime}$ be a homomorphism of affine group schemes over $k$ and let

$$
\tilde{\theta}: \operatorname{Rep}_{k}\left(G^{\prime}\right) \rightarrow \operatorname{Rep}_{k}(G)
$$

be the functor given by sending $\rho^{\prime}: G^{\prime} \rightarrow \mathrm{GL}(V)$ to $\rho^{\prime} \circ \theta: G \rightarrow \operatorname{GL}(V)$. An object $\rho: G \rightarrow \operatorname{GL}(V)$ in $\operatorname{Rep}_{k}(G)$ is said to be a subquotient of an object $\eta: G \rightarrow \mathrm{GL}(W)$ in $\operatorname{Rep}_{k}(G)$ if there are two $G$-submodules $V_{1} \subset V_{2}$ of $W$ such that $V \cong V_{2} / V_{1}$ as $G$-modules. Let $\theta: G \rightarrow G^{\prime}$ be a homomorphism of affine algebraic groups over $k$. Then $\theta$ is a closed immersion if and only if every object of $\operatorname{Rep}_{k}(G)$ is isomorphic to a subquotient of an object of the form $\tilde{\theta}\left(V^{\prime}\right)$, for some $V^{\prime} \in \operatorname{Rep}_{k}\left(G^{\prime}\right)$.

Let $W$ be a numerically flat bundle on $\mathcal{Q}$ which corresponds to a finite dimensional representation $\rho: \pi^{S}(\mathcal{Q}, q) \rightarrow \mathrm{GL}(V)$. We will show that there is a numerically flat bundle $W^{\prime}$ on $S^{d}(C)$ such that $W \cong \phi^{*} W^{\prime}$. This precisely means that there is a representation $\rho^{\prime}: \pi^{S}\left(S^{d}(C), \phi(q)\right) \rightarrow \mathrm{GL}(V)$ such that $\rho=\rho^{\prime} \circ \phi_{*}^{S}$. Now by the criterion in the preceding para we see that $\phi_{*}^{S}$ is a closed immersion. 
By Grauert's theorem [Har77, Corollary 12.9] and Proposition 7.3, it follows that if $W$ is a numerically flat bundle on $\mathcal{Q}$ then $\phi_{*}(W)$ is a locally free sheaf on $S^{d} C$ and the natural map $\phi^{*} \phi_{*}(W) \rightarrow W$ is an isomorphism. It follows easily that $\phi_{*}(W)$ is numerically flat. This is easily checked because given a morphism $f: X \rightarrow Y$ between two projective varieties, and a morphism from a projective curve $C \rightarrow Y$, we can always find a cover $C^{\prime} \rightarrow C$ such that the composite $C^{\prime} \rightarrow Y$ lifts to a map $C^{\prime} \rightarrow X$. This proves that $\phi_{*}^{S}$ is a closed immersion.

From the $S$-fundamental group scheme we recover the Nori fundamental group scheme as the inverse limit of finite quotients. Similarly, the etale fundamental group scheme can be recovered as the inverse limit of finite and reduced quotients. Thus, we get the following corollary. (See $\S 5.5$ in [PS19b] for more details.)

Corollary 7.6. The induced map $\phi_{*}^{N}: \pi^{N}(\mathcal{Q}, q) \rightarrow \pi^{N}\left(S^{d} C, \phi(q)\right)$ is an isomorphism. The induced map $\phi_{*}^{\text {ét }}: \pi^{\text {ét }}(\mathcal{Q}, q) \rightarrow \pi^{\text {ét }}\left(S^{d} C, \phi(q)\right)$ is an isomorphism.

\section{REFERENCES}

[BDH15] Indranil Biswas, Ajneet Dhillon, and Jacques Hurtubise. Brauer groups of Quot schemes. Michigan Math. J., 64(3):493-508, 2015. doi: $10.1307 / \mathrm{mmj} / 1441116655$.

[BH15] Indranil Biswas and Amit Hogadi. On the fundamental group of a variety with quotient singularities. Int. Math. Res. Not. IMRN, (5):1421-1444, 2015. doi:10.1093/imrn/rnt261.

[BKR19] Cristina Bertone, Steven L. Kleiman, and Margherita Roggero. On the quot scheme Quot $\mathcal{O}_{\mathbb{P}^{1} / \mathbb{P}^{1} / k}^{d}, 2019$, arXiv:1906.01953.

[BPS06] Indranil Biswas, A. J. Parameswaran, and S. Subramanian. Monodromy group for a strongly semistable principal bundle over a curve. Duke Math. J., 132(1):1-48, 2006. doi:10.1215/S0012-7094-06-13211-8.

[DMOS82] Pierre Deligne, James S. Milne, Arthur Ogus, and Kuang-yen Shih. Hodge cycles, motives, and Shimura varieties, chapter 2, pages 101-228. SpringerVerlag, Berlin-New York, 1982. doi:10.1007/978-3-540-38955-2_4.

[FGA05] Barbara Fantechi, Lothar Göttsche, Luc Illusie, Steven L. Kleiman, Nitin Nitsure, and Angelo Vistoli. Fundamental algebraic geometry, volume 123 of Mathematical Surveys and Monographs. American Mathematical Society, Providence, RI, 2005. doi:10.1090/surv/123. Grothendieck's FGA explained.

[Har77] Robin Hartshorne. Algebraic geometry. Springer-Verlag, New York-Heidelberg, 1977. Graduate Texts in Mathematics, No. 52.

[HM11] Amit Hogadi and Vikram Mehta. Birational invariance of the $S$-fundamental group scheme. Pure Appl. Math. Q., 7(4, Special Issue: In memory of Eckart Viehweg):1361-1369, 2011.

[Lan11] Adrian Langer. On the S-fundamental group scheme. Ann. Inst. Fourier (Grenoble), 61(5):2077-2119 (2012), 2011. doi:10.5802/aif.2667.

[Lan12] Adrian Langer. On the S-fundamental group scheme. II. J. Inst. Math. Jussieu, 11(4):835-854, 2012. doi:10.1017/S1474748012000011.

[Mat86] Hideyuki Matsumura. Commutative ring theory, volume 8 of Cambridge Studies in Advanced Mathematics. Cambridge University Press, Cambridge, 1986. Translated from the Japanese by M. Reid. 
[Nor76] Madhav V. Nori. On the representations of the fundamental group. Compositio Math., 33(1):29-41, 1976. URL http: //www . numdam.org/item?id=CM_1976_-_33_1_29_0.

[Nor82] Madhav V. Nori. The fundamental group-scheme. Proc. Indian Acad. Sci. Math. Sci., 91(2):73-122, 1982. doi:10.1007/BF02967978.

[PS19a] Arjun Paul and Ronnie Sebastian. Fundamental group schemes of $n$-fold symmetric product of a smooth projective curve, 2019, arXiv:1907.09388.

[PS19b] Arjun Paul and Ronnie Sebastian. Fundamental group schemes of Hilbert scheme of $n$ points on a smooth projective surface, 2019, arXiv:1907.04290.

[Stk] The Stack Project. https://stacks.math.columbia.edu.

Department of Mathematics, Indian Institute of Technology Bombay, Powai, Mumbai 400076, Maharashtra, India.

E-mail address: chandra@math.iitb.ac.in

Department of Mathematics, Indian Institute of Technology Bombay, Powai, Mumbai 400076, Maharashtra, India.

E-mail address: ronnie@math.iitb.ac.in 